Granular superconductivity in metallic and insulating nanocrystalline boron-doped diamond thin films

This article has been downloaded from IOPscience. Please scroll down to see the full text article.

2010 J. Phys. D: Appl. Phys. 43374019

(http://iopscience.iop.org/0022-3727/43/37/374019)

View the table of contents for this issue, or go to the journal homepage for more

Download details:

IP Address: 134.58.253.57

The article was downloaded on 01/12/2011 at 08:29

Please note that terms and conditions apply. 


\title{
Granular superconductivity in metallic and insulating nanocrystalline boron-doped diamond thin films
}

\author{
B L Willems ${ }^{1,2}$, G Zhang ${ }^{1}$, J Vanacken ${ }^{1}$, V V Moshchalkov ${ }^{1}$, \\ S D Janssens ${ }^{3}$, K Haenen ${ }^{3,4}$ and P Wagner ${ }^{3,4}$ \\ ${ }^{1}$ INPAC-Institute for Nanoscale Physics and Chemistry, Katholieke Universiteit Leuven, Celestijnenlaan \\ 200-D, 3000-Leuven, Belgium \\ ${ }^{2}$ Facultad de Ciencias Físicas, Universidad Nacional Mayor de San Marcos, PO 14-0149, Lima-14, Perú \\ ${ }^{3}$ Institute for Materials Research (IMO), Hasselt University, BE-3590 Diepenbeek, Belgium \\ ${ }^{4}$ Division IMOMEC, IMEC vzw, Wetenschapspark 1, BE-3590 Diepenbeek, Belgium \\ E-mail: bramleo@hotmail.com
}

Received 26 February 2010, in final form 27 May 2010

Published 2 September 2010

Online at stacks.iop.org/JPhysD/43/374019

\begin{abstract}
The low-temperature electrical transport properties of nanocrystalline boron-doped diamond (b-NCD) thin films have been found to be strongly affected by the system's granularity. The important differences between the high and low-temperature behaviour are caused by the inhomogeneous nucleation of superconductivity in the samples. In this paper we will discuss the experimental data obtained on several b-NCD thin films, which were studied by either varying their thickness or boron concentration. It will be shown that the low-temperature properties are influenced by the b-NCD grain boundaries as well as by the appearance of an intrinsic granularity inside these granules. Moreover, superconducting effects have been found to be present even in insulating b-NCD films and are responsible for the negative magnetoresistance regime observed at low temperatures. On the other hand, the low-temperature electrical transport properties of b-NCD films show important similarities with those observed for granular superconductors.
\end{abstract}

(Some figures in this article are in colour only in the electronic version)

\section{Introduction}

The discovery of superconductivity in boron-doped diamond [1] adds a new intriguing property to the already broad branch exhibited by these materials. Pure diamond is known to be a wide-gap insulator with a band-gap of $\sim 5.5 \mathrm{eV}$ and can be doped in a controlled way with boron, phosphorus and even nitrogen [2,3]. Among the techniques available for growing doped-diamond samples, plasma-enhanced chemical vapour deposition (PECVD) is of wide use. By seeding silicon or quartz substrates with diamond powder, carbon diffusion is blocked and diamond nucleation sites are generated [4]. When the CVD growth occurs in a hydrogen-rich plasma, an evolution in the diamond grain size from the small crystals at the seeded substrate is observed [5]. The resulting diamond thin films are then consisting of nanocrystalline diamond (NCD) granules.
The observation of superconductivity in PECVD borondoped NCD (b-NCD) thin films was reported for the first time in 2006 [6], two years after its discovery in borondoped diamond [1]. Experimental results so far point out the strong influence of granularity on the low-temperature electrical transport properties of this system [7-10]. According to Mareš et al [7], the structural NCD grain boundaries play a fundamental role in the low-temperature behaviour. An unambiguously proof for the latter would be the fact that the relative width of the superconducting transition, which is measured as $\delta T / T_{\mathrm{C}}$, decreases upon increasing the sample thickness [7]. Here, $\delta T$ is the difference between the on-set and off-set transition temperature and $T_{\mathrm{C}}$ is the off-set temperature. Furthermore, the NCD grains are considered to be electrically connected with each other only in a few spots with characteristic dimensions of $\sim 5 \mathrm{~nm}$ [7]. These spots act as weak links once the system is in the superconducting 
state [7]. Moreover, grain boundaries are also claimed as causing the negative magnetoresistance regime observed in highly nitrogen-doped ultra-nanocrystalline diamond (UNCD) thin films [11].

In addition to its structural granular morphology (see figure 1), b-NCD may also posses an intrinsic granularity with a typical length scale much smaller than the structural b-NCD granules $[9,10]$. The inhomogeneous boron doping of diamond is perhaps one of the most important sources for intrinsic granular effects $[10,12]$. However, recent scanning tunnelling microscopy (STM) measurements give evidence that the b-NCD electronic band structure can also induce an ordered nanosized granularity [13].

Upon increasing the boron concentration above a critical value $n_{\mathrm{C}}$, boron-doped diamond undergoes an insulator-metal transition (IMT). At relatively low concentrations $\left(n_{\mathrm{B}} \approx\right.$ $10^{17} \mathrm{~cm}^{-3}$ ), the boron atoms enter the diamond crystal by substituting carbon atoms and form an acceptor ground level with energy $E_{\mathrm{A}} \approx 370 \mathrm{meV}$ above the host's valence band $[2,14]$. This energy is very close to the ionization energy $\left(E_{\mathrm{i}} \approx 368 \mathrm{meV}\right)$ found from optical experiments [2]. In the effective hole-mass approximation, the IMT in diamond is expected to happen for dopant concentrations of $n_{\mathrm{C}}=$ $\left(0.26 / a_{\mathrm{B}}\right)^{3} \approx 2 \times 10^{20} \mathrm{~cm}^{-3}$, where $a_{\mathrm{B}} \approx 3.5 \AA$ is the boron Borh's radius [2]. Experimentally, the $n_{\mathrm{C}}$ values obtained for boron-doped diamond samples made by PECVD techniques lay between $2 \times 10^{20}$ and $4.5 \times 10^{20} \mathrm{~cm}^{-3}[15,16]$, in agreement with theory. From the above, the inter-boron distance $r_{\mathrm{BB}}$, which is obtained by [17]

$$
r_{\mathrm{BB}}=\left(\frac{3}{4 \pi n_{\mathrm{B}}}\right)^{1 / 3}
$$

should be between 8 and $10 \AA$ when $n_{\mathrm{B}}=n_{\mathrm{C}}$. Since $a_{\mathrm{B}} \approx$ $3.5 \AA$ [2], the metallic regime is then providing percolating paths of overlapped wave functions between neighbouring boron atoms.

So far, supercoductivity has been only observed in metallic boron-doped diamond, i.e. when $n_{\mathrm{B}}>n_{\mathrm{C}}[8,15]$. Furthermore, the critical temperature $\left(T_{\mathrm{C}}\right)$ of superconducting single-crystalline boron-doped diamond has been found to depend on $n_{\mathrm{B}}$ as $T_{\mathrm{C}} \sim\left(n_{\mathrm{B}} / n_{\mathrm{C}}-1\right)^{1 / 2}$ [15], being the highest value obtained $T_{\mathrm{C}}=11.4 \mathrm{~K}$ [18].

Two recent experimental observations strongly support the phonon-mechanism proposed by Bardeen, Schriefer and Cooper (BCS) for the superconductivity in diamond: (1) the superconducting gap of single-crystalline boron-doped diamond shows the BCS temperature evolution [19] and (2) the $T_{\mathrm{C}}$ is dependent on the isotope of the carbon (isotope effect) [20].

In this paper, we discuss on the strong influence of granularity on the low-temperature electrical transport properties of $b-\mathrm{NCD}$ thin films prepared by PECVD methods. The high critical magnetic field values obtained for these systems despite being low- $T_{\mathrm{C}}$ BCS superconductors are explained by an inhomogeneous nucleation of the superconducting order parameter inside the b-NCD granules. This intrinsic granularity has a typical length scale of the order of $<50 \mathrm{~nm}$, a much smaller size than the structural diamond granule of b-NCD films with thicknesses larger than $200 \mathrm{~nm}$. The latter is confirmed by recent STM experiments performed on superconducting b-NCD thin films, in which it has been observed that the superconducting gap varies typically over $30 \mathrm{~nm}$ [9]. On the other hand, the broadening in temperature of the superconducting transition is directly related to the number of grain boundaries, in agreement with Mareš et al [7]. Moreover, we will show that the reduction of this number is accompanied by a weakening of the effect of superconducting fluctuations.

Superconducting effects are not only exclusive to metallic b-NCD, but can also be present when the system is insulating. These effects are detectable with electrical transport experiments by the observation of a negative magnetoresistance regime at low temperatures [10]. We will show that this regime is explained by a competition between inter-grain quasiparticle tunnelling and intra-grain superconducting fluctuations.

In the last part we will also discuss the similarities observed in the low-temperature electrical transport properties of b-NCD and granular superconductors, which consist of metallic granules, such as lead or aluminium, embedded in an insulating matrix.

\section{Experimental}

The b-NCD films were made by PECVD techniques. In short, the sample preparation process can be divided into two main steps $[5,4]$ :

(1) Substrate seeding: this consists in covering a substrate with a layer of diamond powder. First, the size distribution of a commercially available diamond powder is reduced by milling the powder with zirconia beads. This is done in order to obtain small particles $(\lesssim 5 \mathrm{~nm})$ and hence increase the nucleation density. Next, a suspension is formed by mixing the milled powder with water or alcohol, and a wafer is placed into this suspension. After placing the suspension with the wafer into an ultrasonic bath for about $30 \mathrm{~min}$, the wafer is taken out and spun dry. As a result, a seeding monolayer is left on the substrate.

(2) Microwave PECVD growth: the substrate with a seeding layer on top is placed into an ASTex series microwave PECVD reactor and diamond films were grown in a hydrogen rich plasma with low methane concentrations $(<3 \%)$. Typically, the substrate was held at $700^{\circ} \mathrm{C}$ under a pressure of 35 Torr. Boron doping was achieved by injecting trimethylboron (TMB) gas during the whole growth process with the concentrations of $\mathrm{TMB} / \mathrm{CH}_{4}$ ranging between 4000 and 8000 ppm.

For this work, diamond layers were grown on two different substrates: $\mathrm{Si}$ and quartz. The sets of samples investigated were either consisting of films with varying thicknesses or with different boron concentrations $n_{\mathrm{B}}$. The doping level was mainly controlled by the amount of TMB gas injected, and the thickness by tuning the growth time.

The macroscopic properties of the samples were characterized by means of electrical transport measurements. 

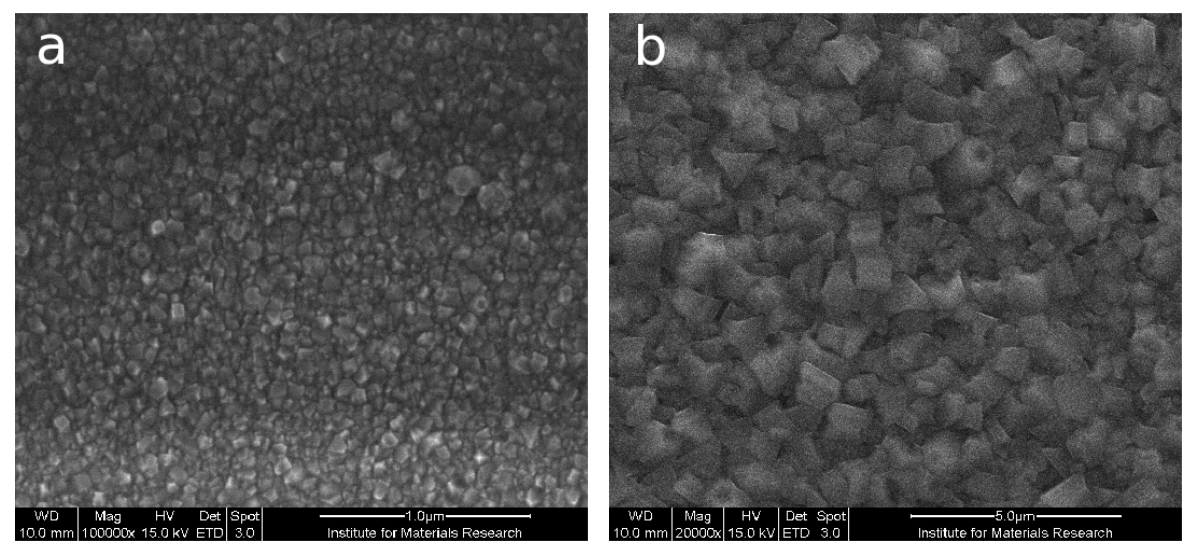

Figure 1. (a) The SEM picture corresponding to a $65 \mathrm{~nm}$ thick film. Here, the scale is $1 \mu \mathrm{m}$ and a typical grain size of $50 \mathrm{~nm}$ can be inferred. (b) A SEM picture of a $800 \mathrm{~nm}$ thick film. The length scale is $5 \mu \mathrm{m}$ and the typical grain size obtained $1 \mu \mathrm{m}$.

These were probed by using a four-terminal configuration with the contacts silver pasted to the corners of the 1 by $1 \mathrm{~cm}^{2}$ square samples. Current is caused to flow along one edge of the sample while the voltage was measured between the extremities of the opposite edge. We have found that the resistance $(R)$ measured by using this configuration showed almost no difference with the obtained when a transport bridge was patterned on one of the samples. The latter evidences the absence of important current re-distribution processes in the whole temperature and field range, and hence the sample's extrinsic (resistance) and intrinsic (resistivity) parameters only differ by a constant. The temperature range between 272 and $1.5 \mathrm{~K}$ was studied in an Oxford ${ }^{4} \mathrm{He}$ cryostat. This setup is provided with a $12 \mathrm{~T}$ dc magnet coil and a Lakeshore temperature controller. Four-point resistivity measurements were performed with a nanovoltmeter (Hewlett Packard 34420A) and a programmable current source (Keithley 220). To eliminate thermal or offset voltages while using the nanovoltmeter, the measured voltage is averaged over both positive and negative polarities of the injected current. Typically, a current of $10 \mu \mathrm{A}$ was used. On the other hand, the temperature range between 1.6 and $0.4 \mathrm{~K}$ was studied in a Heliox ${ }^{3} \mathrm{He}$ cryostat by Oxford, provided with a $5 \mathrm{~T} \mathrm{dc}$ magnet coil and a Lakeshore temperature controller as well. In this setup, the samples resistance was measured by lock-in techniques. Typically, the values for the current and frequency were $10 \mu \mathrm{A}$ and $77 \mathrm{~Hz}$, respectively. In all measurements, fields were applied perpendicularly to the sample surface.

\section{Sample morphology}

All the samples show a granular morphology which is revealed by scanning electron microscope (SEM) imaging, as can be seen in figures $1(a)$ and $(b)$. Figure $1(a)$ corresponds to a $65 \mathrm{~nm}$ thick b-NCD film, in which a typical lateral size of $50 \mathrm{~nm}$ has been inferred for the diamond granules. For the case of an $800 \mathrm{~nm}$ thick b-NCD film (figure $1(b)$ ), the typical lateral grain size is around $1 \mu \mathrm{m}$. We have found a more-or-less linear correspondence between sample thickness and lateral grain size in our films, and both parameters were mainly controlled by the growth time, i.e. the time spent in the CVD reactor. This correspondence is characteristic of the van der Drift growth regime [21]. The way the diamond grains grow obey the maxim 'survival of the largest' [5]: the nuclei with their fastest growing orientation perpendicular to the surface will overgrow the slower facets as the film coalesces, forming heavily twinned agglomerates. XRD analysis shows the presence of (2 20$)$ and (1 11 1) peaks, confirming the polycrystalline nature of the films.

In what follows, the samples grown on $\mathrm{Si}$ and quartz substrates will be labelled as s and q, respectively.

\section{Granular superconductivity}

Figure 2 shows the resistance versus temperature dependence $(R(T))$ of a set of $200 \mathrm{~nm}$ thick b-NCD films grown onto quartz substrates under different TMB concentrations. Samples q1 and q2 show their $R(T)$ dependences typical of an insulator as can be seen in the inset of figure 2. By re-plotting $R(T)$ as $\ln (R)$ versus $T^{-\alpha}$, good fits were obtained for $\alpha=1$ [10], indicating that electrical transport mainly occurs by nearest neighbour hopping processes [22].

On the other hand, samples $\mathrm{q} 3$, q4 and q5 show their $R(T)$ to increase upon lowering the temperature as $R \sim$ $\ln (T)$, a typical dependence for metallic boron-doped diamond [22]. Moreover, a superconducting transition is observed for samples $\mathrm{q} 4$ and $\mathrm{q} 5$ with $T_{\mathrm{C}}$ equal to $1.8 \mathrm{~K}$ and $2.2 \mathrm{~K}$, respectively. The latter $T_{\mathrm{C}}$ was obtained by taking the temperature at which the $R(T)$ takes the half value of the normal resistance.

From the above, it is clear that figure 2 shows an IMT when going from $\mathrm{q} 1$ to $\mathrm{q} 5$. Previous studies on b-NCD thin films grown under similar conditions and in the same CVD reactor as the ones presented here, and whose $n_{\mathrm{B}}$ was obtained by secondary ion mass spectroscopy analysis [16]; confirm that the observed IMT is driven by varying $n_{\mathrm{B}}$. In other words, $n_{\mathrm{B}}$ increases systematically when going from sample q1 to q5.

It is important to note that the increase in the $R(T)$ of all the samples is rather slow down to $4 \mathrm{~K}$, approximately. Below this temperature, an important departure, either upwards or downwards, is observed, suggesting the presence of superconducting grains in the samples. 


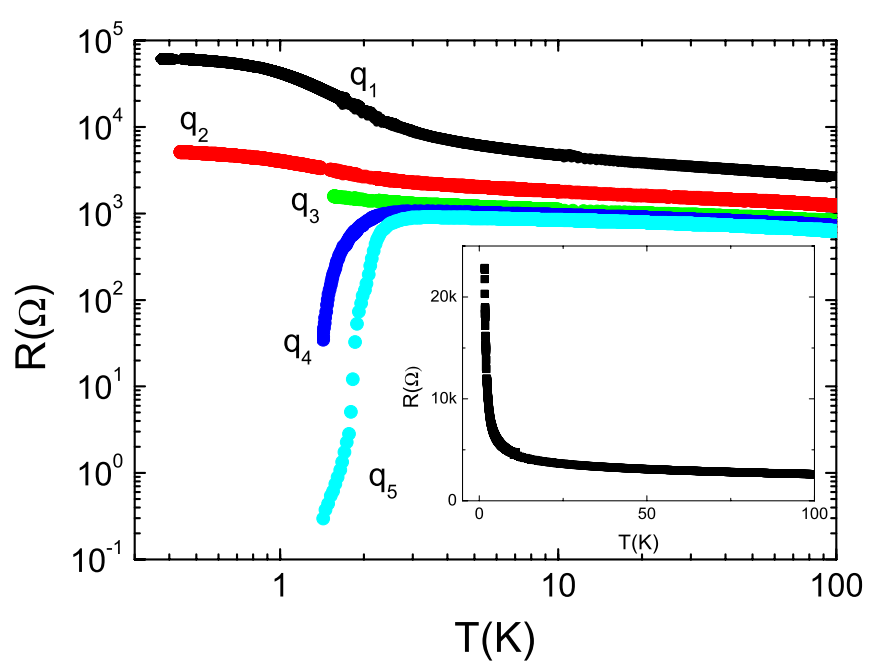

Figure 2. The temperature dependence of the resistance (plotted on a log-log scale) of a set of $200 \mathrm{~nm}$ thick b-NCD films grown on quartz substrates. The samples differ from each other in their boron concentration, which increases when going from q1 to q5. The electrical transport properties of samples q1 and q2 were measured down to $0.4 \mathrm{~K}$ while of the other samples down to $1.5 \mathrm{~K}$. The $T_{\mathrm{C}}$ of $\mathrm{q} 4$ and $\mathrm{q} 5$ are $1.8 \mathrm{~K}$ and $2.2 \mathrm{~K}$, respectively. (Inset) The $R(T)$ dependence of sample q1 plotted on a linear scale. The resistance is shown to be incremented in approximately one order of magnitude between 10 and $0.4 \mathrm{~K}$.

\subsection{Superconducting $b-N C D$ thin films}

For the case of heavily doped b-NCD films, the following characteristics have been observed for their $R(T)$ dependence:

(1) A broad transition in temperature towards a state of zero resistance, a characteristic of granular superconductivity $[8,23]$.

(2) The broadening of the transition increases upon applying an external magnetic field (see figure 4).

(3) $T_{\mathrm{C}}<4 \mathrm{~K}$ when taking the half value of the normal resistance as criterion.

(4) The $R(T)$ curves measured under zero and $12 \mathrm{~T}$ magnetic fields split typically at temperatures below $8 \mathrm{~K}$ (see figure 4).

(5) The samples reveal an important increase in their diamagnetic response at temperatures two to three times $T_{\mathrm{C}}$ when measured in a SQUID magnetometer [9].

This last point is a clear proof for the inhomogeneous boron doping of the samples. Hence, for $T>T_{\mathrm{C}}$ highly doped regions become superconducting and their presence is detected by magnetic measurements. Next, a state of zero resistance is achieved below $4 \mathrm{~K}$ once percolating paths of Josephsoncoupled regions are provided.

Table 1 show some parameters corresponding to two sets of b-NCD thin films grown onto $\mathrm{Si}$ substrates. In this, the $\mathrm{TMB} / \mathrm{CH}_{4}$ ratio used during the CVD growth is shown together with the sample thickness, the critical temperature $T_{\mathrm{C}}$, the second critical magnetic field $B_{\mathrm{C} 2}(0)$ and the GinzburgLandau zero temperature coherence length $\xi(0)$. The $B_{\mathrm{C} 2}(0)$ values were obtained from the linear fits performed on the $T_{\mathrm{C}}$ versus magnetic field plots. On the other hand, $\xi(0)$ was
Table 1. Growth and superconducting parameters of b-NCD thin films with varying thicknesses/boron doping.

\begin{tabular}{lllllc}
\hline Sample & $\begin{array}{l}\mathrm{TMB} / \mathrm{CH}_{4} \\
(\mathrm{ppm})\end{array}$ & $\begin{array}{l}\text { Film thickness } \\
(\mathrm{nm})\end{array}$ & $\begin{array}{l}T_{\mathrm{C}} \\
(\mathrm{K})\end{array}$ & $\begin{array}{l}B_{\mathrm{C} 2}(0) \\
(\mathrm{T})\end{array}$ & $\begin{array}{l}\xi(0) \\
(\mathrm{nm})\end{array}$ \\
\hline s1 & 6500 & 65 & $<0.4$ & - & - \\
s2 & 6500 & 85 & 1.6 & 2.7 & 11 \\
s3 & 6500 & 200 & 2.78 & 4.3 & 8.7 \\
s4 & 6500 & 490 & 2.9 & - & - \\
s5 & 6500 & 800 & 3.1 & 4.1 & 8.9 \\
s6 & 6000 & 200 & 2.78 & 4.3 & 8.7 \\
s7 & 5500 & 200 & 2.71 & 3.9 & 9.2 \\
s8 & 5000 & 200 & 2.37 & 4 & 9.1 \\
s9 & 4800 & - & 2 & - & - \\
\hline
\end{tabular}

derived from the Ginzburg-Landau expression for $B_{\mathrm{C} 2}(0)$ [24]

$$
B_{\mathrm{C} 2}=\frac{\Phi_{0}}{2 \pi \xi^{2}},
$$

where $\Phi_{0}$ is the magnetic flux quanta.

Samples s1 to s5 (set $\boldsymbol{A}$ ) only differ in the growth time while s6 to s9 (set $\boldsymbol{B}$ ) in the $\mathrm{TMB} / \mathrm{CH}_{4}$ ratio used. It is important to mention that by performing a qualitative analysis on the secondary ion mass spectroscopy data of samples s1, s3 and s5, similar values were obtained for their boron/carbon ratio, confirming that these films have approximately equal $n_{\mathrm{B}}$ [25].

Figure $3(a)$ shows the evolution of the $T_{\mathrm{C}}$ on the $\mathrm{TMB} / \mathrm{CH}_{4}$ ratio used during the growth process of the samples corresponding to set $\boldsymbol{B}$. Based on the fact that the $T_{\mathrm{C}}$ of borondoped diamond depends strongly on $n_{\mathrm{B}}$ [15], we can conclude that the $\mathrm{TMB} / \mathrm{CH}_{4}$ ratio controls the amount of boron doping $\left(n_{\mathrm{B}}\right)$ in the samples, as long as the other growth parameters are kept constant. On the other hand, the data obtained for the samples of set $\boldsymbol{A}$ show a systematic increase in $T_{\mathrm{C}}$ with the film thickness. This is shown in figure $3(b)$.

In figure 4 a semi-logarithmic plot of the $R(T)$ dependences of samples s2 and s5 is shown for different applied magnetic fields. It can be seen that the thinner the film is, the broader its superconducting transition in temperature. Moreover, this broadening is further enhanced upon applying magnetic fields.

In the normal state, the resistance of samples s2 and s5 shows a logarithmic dependence on the temperature

$$
R=R_{0} \ln \left(T_{0} / T\right)
$$

where $R_{0}$ and $T_{0}$ are constant (see figure 4). This is a general feature for all metallic b-NCD thin films studied. Furthermore, it has been observed that $R_{0}$ becomes smaller upon increasing the sample thickness. Hall experiments show that the logarithmic temperature dependence is accompanied by a slow decrease in the hole concentration when the system is cooled down [25].

A $R \sim \ln (T)$ dependence is often attributed to twodimensional (2D) weak localization (WL) effects stemming from coherent backscattering processes on impurities [26, 27]. WL effects are expected to settle in for metallic doped-diamond films because of the high degree of disorder present in these 

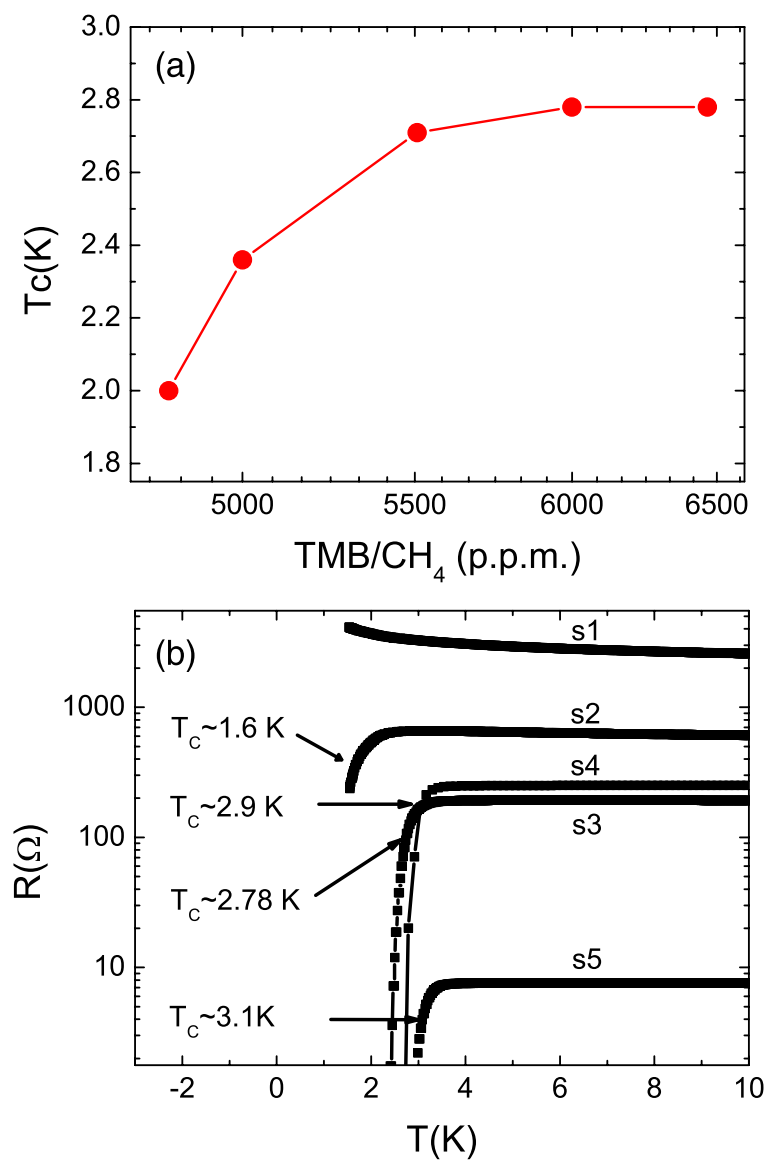

Figure 3. (a) The evolution of the $T_{\mathrm{C}}$ of the samples with the same thickness and grain size, as a function of the TMB concentration. (b) The $R(T)$ curves of the samples with varying thicknesses plotted on a semi-logarithmic scale. Here, the corresponding $T_{\mathrm{C}}$, defined as the temperature at which the resistance, takes the half value of the normal one, are indicated.

materials $[8,15]$. The interplay between the temperature dependence of the sample resistivity and of the carrier concentration fits into the WL scenario. Furthermore, it has been observed that for sample thicknesses above $200 \mathrm{~nm}$ the resistance is incremented in less than $10 \%$ between 30 and $1.5 \mathrm{~K}$, in agreement to what is expected when WL effects are present [28]. However, a negative magnetoresistance (negative-MR), a conclusive proof for the existence of 2D-WL processes [27], was not resolved for any of the samples when magnetic fields were applied perpendicularly. This leaves the possibility open for the contribution from non-WL effects, to which a $R \sim \ln (T)$ dependence is also attributed. These not yet understood mechanisms are claimed to govern the electrical properties of some granular superconducting samples in the vicinity of the superconductor insulator transition [29]. Moreover, samples thinner than $100 \mathrm{~nm}$ show their resistance incremented up to $60 \%$ over the last temperature decade before undergoing the superconducting transition, a value no longer consistent with WL regimes [28].

\subsection{Superconducting effects in insulating $b-N C D$ films}

Superconducting grains have been also found to be present in insulating b-NCD samples located close to the IMT.

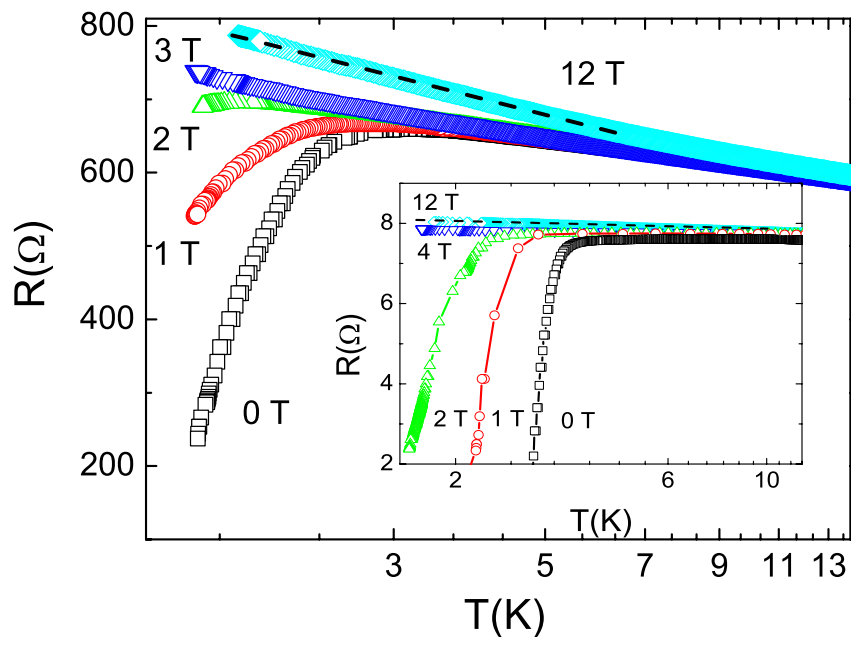

Figure 4. The $R(T)$ curves, measured under different applied fields (oriented perpendicularly), corresponding to the films with thicknesses $85 \mathrm{~nm}$ (s2) and (inset) $800 \mathrm{~nm}$ (s5). Note that the splitting between the zero and $12 \mathrm{~T}$ field curves occurs below $8 \mathrm{~K}$ for both samples.

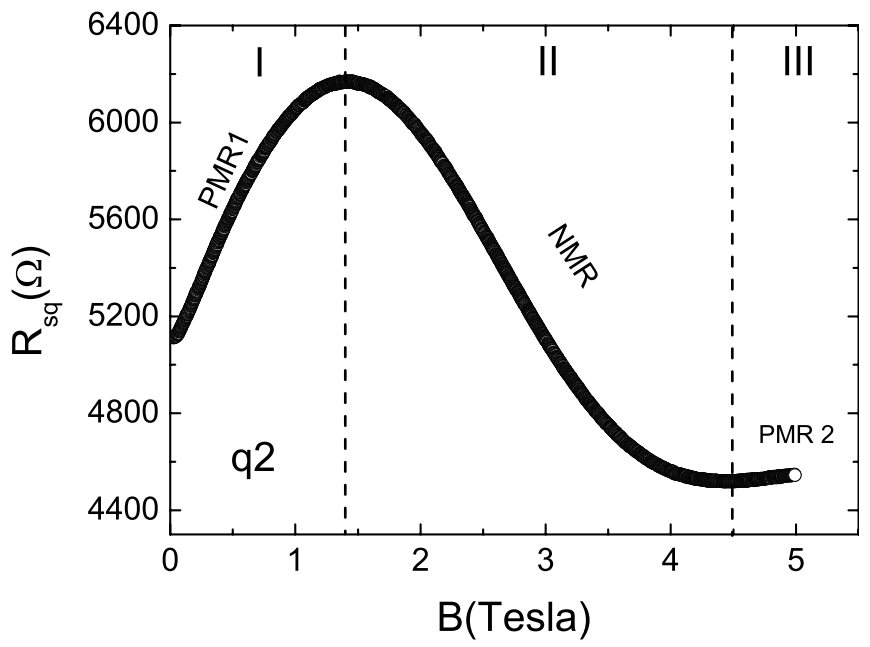

Figure 5. The $R(B)$ isotherm of sample $\mathrm{q} 2$, taken at $0.5 \mathrm{~K}$. In region I some of the grains Josephson couple with each other while in II superconducting fluctuations only occur inside the grains. In region III, superconductivity is totally absent and the PMR is caused by orbital shrinking.

Furthermore, their presence is detectable under electrical transport measurements by the observation of a negative-MR regime at low temperatures [10]. Such a regime was measured in the samples q1 and q2 of figure 2.

Upon increasing the applied magnetic field, the samples $\mathrm{q}_{1}$ and $\mathrm{q}_{2}$ show positive magnetoresistance $(\mathrm{PMR}) \rightarrow$ negative-MR $\rightarrow$ PMR crossovers. The latter can be seen in figure 5 where the magnetoresistance $(R(B))$ of $\mathrm{q}_{2}$, measured at $0.5 \mathrm{~K}$, is shown. To distinguish between the two PMR regimes, the first will be referred as PMR1 (region I in figure 5) and that recovered at high fields as PMR2 (region III). The same dependences have been found for sample $q 1$. However, there are some differences: (1) the negative-MR regime appears at higher temperatures and is more significant for q1 and (2) q2 shows a stronger PMR1 signal with a positive variation in the 


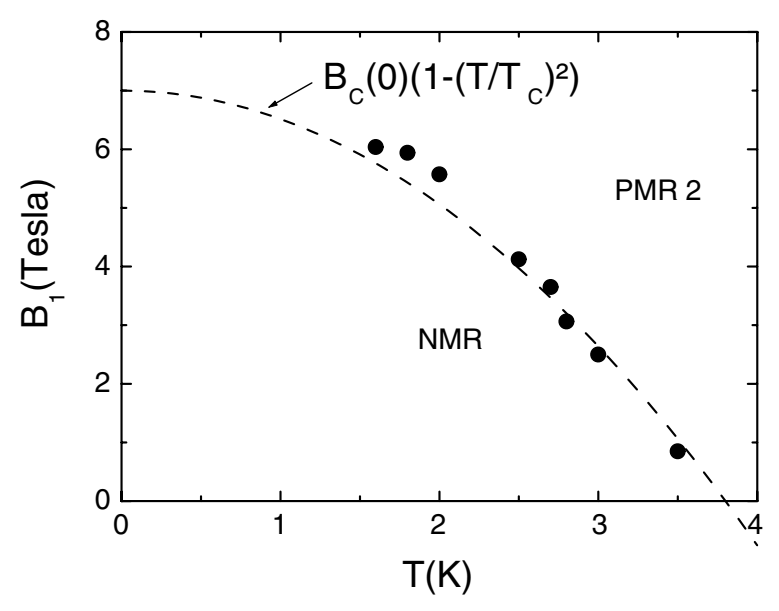

Figure 6. The temperature dependence of the fields at which the NMR $\rightarrow$ PMR 2 crossover happens for sample q1. The dashed line corresponds to the fit of the $B_{1}$ values by using a temperature dependence similar to that empirically found for the superconducting critical field $B_{\mathrm{C}}$ when $T_{\mathrm{C}}=4 \mathrm{~K}$.

resistance $\sim 20 \%$ at the lowest measured temperature [10]. A comparative analysis with the transport data (figure 2) shows a positive correlation between the strength of the negative-MR signal, the temperature at which it appears and the magnitude of the increase in $R(T)$. The crossover from negative-MR to PMR2 at high fields can be attributed to orbital shrinking processes which decouple overlapped wave functions between dopants [22].

A negative-MR regime was previously observed in highly nitrogen-doped UNCD [11], which was analysed within the framework of a WL [11] and a strong localization scenario [30]. Here, we claim that the observed negative-MR in b-NCD samples is caused by the presence of superconducting grains in the insulating films. The proof for this claim is given by the large PMR1 signals observed, which cannot be explained by considering WL nor strong localization effects [10]. An additional support for the presence of superconducting effects in the insulating samples is given in figure 5 . In this, the field values $\left(B_{1}\right)$ at which the crossover between the negative-MR and the PMR2 happens are plotted as a function of the temperature for sample $q_{1}$. It can be seen that these follow a temperature dependence similar to the empirical $B_{\mathrm{C}} \approx B_{\mathrm{C} 0}\left(1-\left(T / T_{\mathrm{C}}\right)^{1 / 2}\right)$ found for the critical field in superconductors [24] (figure 6). Moreover, the $T_{\mathrm{C}}$ extracted from this fit is $3.8 \mathrm{~K}$, a value that is consistent with the temperature at which the zero-field resistance of q1 starts to increase more dramatically (see figure 2).

\section{Discussion}

Metallic b-NCD thin films show a broad superconducting transition in temperature and this broadening is further enhanced upon applying a magnetic field. On the other hand, the increase in the sample thickness, and hence the b-NCD grain size, gives as a result a steeper transition in temperature towards a state of zero resistance. These features can be explained by means of the effect of the structural
Table 2. The evolution of the strength of superconducting fluctuations $(Q)$ upon increasing the film thickness.

\begin{tabular}{lcll}
\hline Sample & $\begin{array}{l}\text { Film thickness } \\
(\mathrm{nm})\end{array}$ & $\begin{array}{l}T_{\mathrm{C}} \\
(\mathrm{K})\end{array}$ & $Q=R_{\text {eff }} / R_{\mathrm{Q}}$ \\
\hline s2 & 85 & 1.6 & 0.6 \\
s3 & 200 & 2.78 & 0.5 \\
s5 & 800 & 3.1 & 0.1 \\
\hline
\end{tabular}

grain boundaries on the low-temperature electrical transport properties [7]. Assuming that the b-NCD grains are electrically connected with each other only in a few spots [7], the amount of inter-grain contacts will be inversely proportional to the grain size, i.e. the bigger the granules, the smaller the number of contacts. At $T<T_{\mathrm{C}}$, the system can then be regarded as consisting of superconducting b-NCD granules connected with each other through weak links which carry supercurrents between Josephson-coupled grains [31]. Within this scenario, a state of zero resistance will be achieved once percolating paths of Josephson-coupled granules are formed.

As is known, the critical current $I_{\mathrm{C}}$, which in this case is the maximum amount of supercurrent that can pass through a weak link, is a measure of how strongly two superconducting grains are coupled [24]. In its turn, $I_{\mathrm{C}}$ decreases with temperature, magnetic field and inter-grain distance while it increases with the cross-sectional area of the weak link [24]. By considering a constant inter-grain distance for all samples, the broadening in temperature of the superconducting transition will then depend on the dispersion of the distribution function for the weak link cross-sectional areas. The mechanism would be the following: first, Josephson coupling between grains will occur for weak links with larger cross-sectional areas and, as a result, clusters along which the current can flow without dissipation are formed. This is manifested by the lowering of the resistance from its maximum value. As temperature goes further down, Josephson coupling becomes possible through weak links with smaller cross-sectional areas, and this will ultimately lead to the opening of percolating superconducting paths.

The fact that the superconducting transition is broader in temperature in thinner films can be interpreted by a lower value for the average inter-grain $I_{C}$. The latter is supported by analysing the effect of the superconducting fluctuations on the low-temperature electrical transport properties. These fluctuations become stronger upon isolating small grains [24], which in our case would be equivalent to making the Josephson coupling between the NCD granules weaker. In table 2, the strength of superconducting fluctuations, which is measured by $Q=R_{\text {eff }} / R_{\mathrm{Q}}$ [32], is shown for three samples with different thicknesses. Here, $R_{\mathrm{Q}}=\hbar / e^{2} \approx 4.1 \mathrm{k} \Omega$ is the quantum resistance and $R_{\text {eff }}=\rho_{\mathrm{N}} / s, \rho_{\mathrm{N}}$ denoting the normal resistivity and $s$ the relevant length scale for the fluctuation [32]. Furthermore, for the estimation of the $Q$ values, $\rho_{\mathrm{N}}$ has been taken as the maximum value for $\rho(T)$ and $s$ as $\xi(0)$ [33].

It can be seen that $Q$ decreases with the film thickness, indicating a stronger coupling between superconducting grains when the number of connecting spots is reduced.

On the other hand, an applied magnetic field will result in a weakening of the inter-grain Josephson coupling. 
As a consequence, the broadening in temperature of the superconducting transition is enhanced.

The samples show rather high values for their $B_{\mathrm{C} 2}(0)$ despite the fact that they are low- $T_{\mathrm{C}}$ BCS superconductors (see table 1). This observation leads us to consider confinement effects in the b-NCD granules. As is known, $B_{\mathrm{C} 2}$ can have values even orders of magnitude higher than those for the bulk sample when superconducting grains are reduced below their bulk magnetic penetration depth [34].

In order to estimate the size of the 'effective' superconducting grains, we consider these as spherical. The magnetic field required for breaking a Cooper-pair by diamagnetic effects can then be related to the grain radius $r$ by [35]

$$
B_{\text {orb }} \approx \frac{\Phi_{0}}{r^{2} \sqrt{g_{\text {dim }}}} \mathrm{T},
$$

where $g_{\mathrm{dim}}=E_{\mathrm{Th}} / d$ the dimensionless conductance, $E_{\mathrm{Th}}$ and $d$ the grain's Thouless energy and the mean energy level spacing, respectively.

In the free-electron approximation $d$ is given by [35]

$$
d=\frac{1.5}{k_{\mathrm{F}} \mathrm{Vol}} \mathrm{eV} \mathrm{nm}^{2},
$$

where $k_{\mathrm{F}}$ is the Fermi vector and $\mathrm{Vol} \sim r^{3}$ the grain volume.

On the other hand, the expression for $E_{\mathrm{Th}}$ in the diffusive regime reads [35]

$$
E_{\mathrm{Th}}=\frac{0.25 k_{\mathrm{F}} \ell}{r^{2}} \mathrm{meV} \mathrm{nm}^{2},
$$

where $\ell$ is the transport mean free path.

By taking the values for $\ell=1.3 \mathrm{~nm}$ [19] and $k_{\mathrm{F}}=$ $4.3 \mathrm{~nm}^{-1}$ [7], a $B_{\text {orb }} \approx 4 \mathrm{~T}$ corresponds to a grain radius $r \approx 36 \mathrm{~nm}$. This implies that the samples with thicknesses above $200 \mathrm{~nm}$ show an intra-granularity with length scale of the order of $30 \mathrm{~nm}$, a value that is consistent with recent STM experiments performed on b-NCD thin films [9]. On the other hand, the expected radius for sample s2 is $43 \mathrm{~nm}$, of the order of its structural grain size.

In the case of the insulating samples, the observation of a PMR1 regime in figure 4, together with the reduction in its magnitude when decreasing $n_{\mathrm{B}}$, can be explained by a diluted distribution of superconducting grains, some of which will start to become Josephson-coupled upon lowering the temperature. The latter leads to the opening of non-percolating zeroresistance channels and, as a result, the sample conductivity experiences a small but measurable increase below the $T_{\mathrm{C}}$ of the grains. Therefore, when switching on the field, $R$ first increases due to the decoupling between the grains (PMR1). Because the $n_{\mathrm{B}}$ of sample $\mathrm{q}_{2}$ is larger than that of $\mathrm{q}_{1}$, more superconducting grains are expected in the former and hence a more significant PMR1 signal. This is in agreement with the experiment.

The negative-MR can be understood as follows: for sufficiently high magnetic fields Cooper-pair transfer between the superconducting grains is suppressed and singlequasiparticle tunnelling settles in [36]. It is well known that the presence of virtual Cooper-pairs (superconducting fluctuations) will affect the density of states (DOS) of the tunnelling quasiparticles since there are two quasiparticles less everytime a Cooper-pair is formed [36]. Hence, intra-grain superconducting fluctuations introduce an additional term in the (inter-grain) conductivity

$$
\sigma \rightarrow \sigma+\Delta \sigma_{\mathrm{DOS}}
$$

where $\Delta \sigma_{\mathrm{DOS}}$ is negative. Therefore, suppression of the virtual Cooper-pairs $\left(\Delta \sigma_{\text {DOS }} \rightarrow 0\right.$ ) by further increasing the field is translated into a decrease in the resistance [36] (NMR in figure 5). The fact that the negative-MR is more significant in $\mathrm{q}_{1}$ can be attributed to the smaller sizes of the regions which are optimally doped, which is analogous to having smaller superconducting grains. Since fluctuations become more important upon reducing the superconductor size [24], higher fields are required for destroying the virtual Cooperpairs inside these grains.

Finally, we would like to remark on the similarities existing in the low-temperature electrical transport properties of b-NCD and films consisting of superconducting granules embedded in an insulating matrix [37-39]. The latter systems undergo an IMT tuned by the inter-grain distance [37-39]. Since b-NCD exhibits an intrinsic granularity, which consists of optimally doped regions with sizes of the order of $<50 \mathrm{~nm}$, the sample $n_{\mathrm{B}}$ will be directly proportional to the granule/total volume ratio $V_{\mathrm{g}} / V_{\mathrm{T}}$. The IMT observed in figure 2 can then be explained as being driven by tuning the $V_{\mathrm{g}} / V_{\mathrm{T}}$ ratio, which is equivalent to varying the average distance between the grains with local $n_{\mathrm{B}}>n_{\mathrm{C}}$.

On the other hand, Cooper-pairs have been found to be present on both insulating and superconducting sides of the IMT of granular superconductors and appear at the $T_{\mathrm{C}}$ corresponding to the bulk value [40,41]. The presence of superconducting fluctuations is evidenced in $R(T)$ curves by the on-set of the resistance decrease/increase at $T=T_{\mathrm{C}}$ which is in its turn strongly affected and/or suppressed by applying an external magnetic field [23]. The analysis of the lowtemperature behaviour of the b-NCD films also show these characteristics (see figure 2).

A $R \sim \operatorname{Ln}(T)$ dependence has been also found for granular superconductors close to the IMT [29]. Furthermore, as for the case of b-NCD, the reduction in $R_{0}$ in equation (3) is also accompanied by a weakening of the effect of superconducting fluctuations [29].

\section{Conclusions}

In this paper, the electrical transport properties of b-NCD thin films at low temperatures have been presented and discussed. It has been found that these systems reveal an intrinsic granularity with a length scale of the order of $<50 \mathrm{~nm}$, which explains the high values of the critical magnetic fields exhibited. The lowtemperature behaviour of the b-NCD films strongly depends on the grain boundaries as well as on the intrinsic granularity. On the other hand, insulating b-NCD thin films have shown the presence of superconducting effects by revealing a negative magnetoresistance regime at low temperatures. Finally, the similarities existing in the electrical transport properties of granular superconductors and b-NCD films are discussed. 


\section{Acknowledgments}

This work was financially supported by the Research Programs G.0068.07 and G.0430.07 of the Research FoundationFlanders (FWO), the Methusalem 'NANO network AntwerpHasselt', the European Science Foundation (ESF), NES programme, the IAP-P6/42 project 'Quantum Effects in Clusters and Nanowires', GOA and FWO projects.

\section{References}

[1] Ekimov E A, Sidorov V A, Bauer E D, Mel'nik N N, Curro N J, Thompson J D and Stishov S M 2004 Nature (London) 428542

[2] Thonke K 2003 Semicond. Sci. Technol. 18 S20-6

[3] Haenen K, Lazea A, Barjon J, DHaen J, Habka N, Teraji T, Koizumi S and Mortet V 2009 J. Phys.: Condens. Matter 21 36364204

[4] Williams O A, Douhéret O, Daenen M, Haenen K, Osawa E and Takahashi M 2007 Chem. Phys. Lett. 4457255

[5] Williams O A, Nesladek M, Daenen M, Michaelson S, Hoffman A, Osawa E, Haenen K and Jackman R B 2008 Diamond Relat. Mater. 171080

[6] Nesládek M, Thompson D, Mer C, Bergonzo P, Hubík P and Mareš J J 2006 Appl. Phys. Lett. 88232111

[7] Mareš J J, Hubík P, Krištofik J and Nesládek M 2008 Phys. Status Solidi a 2052163

[8] Mareš J J, Hubík P and Krištofik J 2007 Diamond Relat. Mater. 16921

[9] Willems B L et al 2009 Phys. Rev. B 80224518

[10] Willems B L, Zhang G, Vanacken J, Moshchalkov V V, Janssens S D, Williams O A, Haenen K and Wagner P 2009 J. Appl. Phys. 106033711

[11] Mareš J J, Hubík P, Krištofik J, Kindl D, Fanta M, Nesládek M, Williams O and Gruen D M 2006 Appl. Phys. Lett. 88092107

[12] Nishizakia T, Takanob Y, Nagaob M, Takenouchic T, Kawaradac H and Kobayashia N 2008 J. Phys. Chem. Solids 693027

[13] Altfeder I B, Hu J J, Voevodin A A and Krim J 2009 Phys. Rev. Lett. 102136104

[14] Mamin R F and Inushima T 2001 Phys. Rev. B 63033201

[15] Klein T et al 2007 Phys. Rev. B 75165313

[16] Gajewski W, Achatz P, Williams O A, Haenen K, Bustarret E, Stutzmann M and Garrido J A 2009 Phys. Rev. B 79045206
[17] Sato T, Ohashi K, Sugai H, Sumi T, Haruna K, Maeta H, Matsumoto N and Otsuka H 2000 Phys. Rev. B 6112970

[18] Takano Y, Takenouchi T, Ishii S, Ueda S, Okutsu T, Sakaguchi I, Umezawa H, Kawarada H and Tachiki M 2007 Diamond Relat. Mater. 16911

[19] Sacepe B, Chapelier C, Marcenat C, Kačmarčik J, Klein T, Bernard M and Bustarret E 2006 Phys. Rev. Lett. 96097006

[20] Ekimov E A, Sidorov V A, Zoteev A V, Lebed J B, Thompson J D and Stishov S M 2008 Sci. Technol. Adv. Mater. 9044210

[21] van der Drift A 1967 Philips Res. Rep. 22267

[22] Shklovskii B I and Efros A L 1984 Electronic Properties of Doped Semiconductors (Springer Series in Solid-State Sciences vol 45) (Germany: Springer)

[23] Beloborodov I S, Lopatin A V, Vinokur V M and Efetov K B 2007 Rev. Mod. Phys. 79469

[24] Tinkham M 1996 Introduction to Superconductivity (Singapore: Mc Graw-Hill)

[25] Willems B L 2009 PhD Thesis Katholieke Universiteit Leuven ISBN 978-90-8649-236-7

[26] Mott N F 1990 Metal-Insulator Transitions (London: Taylor and Francis)

[27] Abrikosov A A 1988 Fundamentals of the Theory of Metals (Amsterdam: North-Holland)

[28] Kobayashi S 1985 Localization, Interaction, and Transport Phenomena ed B Kramer et al (Berlin: Springer) p 18

[29] Simon R W, Dalrymple B J, Van Vechten D, Fuller W W and Wolf S A 1987 Phys. Rev. B 361962

[30] Choy T C, Stoneham A M, Ortuño M and Somoza A M 2008 Appl. Phys. Lett. 92012120

[31] Likharev K K 1984 Dynamics of Josephson Junctions and Circuits (France: Gordon and Breach Science Publishers)

[32] Blatter G, Ivlev B, Kagan Y, Theunissen M, Volokotin Y and Kes P 1994 Phys. Rev. B 5013013

[33] Bustarret E, Kačmarčik J, Marcenat C, Gheeraert E, Cytermann C, Marcus J and Klein T 2004 Phys. Rev. Lett. 93237005

[34] Li W H, Yang C C, Tsao F C and Lee K C 2003 Phys. Rev. B 68184507

[35] Von Delft J and Ralph D C 2001 Phys. Rep. 34561

[36] Belobodorov I S and Efetov K B 1999 Phys. Rev. Lett. 823332

[37] Jaeger H M, Haviland D B, Orr B G and Goldman A M 1989 Phys. Rev. B 40182

[38] Shapira Y and Deutscher G 1983 Phys. Rev. B 274463

[39] Kagawa K, Inagaki K and Tanda S 1996 Phys. Rev. B 53 R2979

[40] Paalanen M A, Hebard A F and Ruel R R 1992 Phys. Rev. Lett. 691604

[41] Strelniker Y M, Frydman A and Havlin S 2007 Phys. Rev. B $\mathbf{7 6} 224528$ 